\title{
Fixed Point Theorem in Fuzzy Metric Spaces
}

\author{
Rajesh Kr. Singh ${ }^{1} \&$ Dr. M. Z. Alam ${ }^{2}$ \\ ${ }^{1}$ Research Scholar, P.G. Dept. of Mathematics, College of Commerce, Arts \& Science, Patna-20. \\ ${ }^{2}$ Associate Professor \& Head, P.G. Dept. of Mathematics, College of Commerce, Arts \& Science,Patna-20.
}

\begin{abstract}
In this paper, we studied the fuzzy metric space as defined by Z.Q.Xia, and F.F.Guo. Defined in different way in the sense of fuzzy scalars instead of fuzzy numbers or real numbers are used to define fuzzy metric. It is proved that every metric space can induce a fuzzy metric space that is complete whenever, the original one is complete. We define fuzzy contraction mapping and try to established fixed point theorem in fuzzy metric space.
\end{abstract}

Key Words: Fuzzy metric space, induced fuzzy metric space, Contraction mapping in fuzzy metric space.

\section{Introduction}

To define fuzzy metric space is of course a problem in fuzzy mathematics which can be used in fuzzy optimization and pattern recognition. There are various approaches to define fuzzy metric space. Some other researcher like Kaleva (1980),George (1994), Gregory (2000),etc. They are using real numbers to measure the distance between fuzzy sets. The problem is that they are using different measure in different problems in fuzzy environment. There does not exist a uniform measure that can be used in all kinds of fuzzy environment. In this paper, an attempt has been made to using fuzzy scalars (fuzzy points defined on the real-valued space R) to measure the distance between fuzzy points which is consistent with the theory of fuzzy linear spaces in the sense of Xia and Guo (2003),more similar to the classic metric spaces. Using Xia and Guo, definition of fuzzy metric space, We established some other results and prove fixed point theorem in fuzzy metric space.

\section{Preliminaries}

2.1 FUZZY POINTS: A fuzzy set in $X$ is called a fuzzy points iff it takes the value ' 0 ' for $y \in X$, except one say $\mathrm{x} \in \mathrm{X}$. If its value at $\mathrm{x}$ is $\lambda(0<\lambda \leq 1)$. We denote this fuzzy point by $x_{\lambda}$, where the point $\mathrm{x}$ is called its support.

$$
x_{\lambda}(y)=\left\{\begin{array}{lll}
\lambda & \text { if } & x=y \\
0 & \text { if } & x \neq y
\end{array}\right.
$$

In this paper, fuzzy points are usually denoted by $(x, \lambda)$, and the set of all fuzzy points defined on $\mathrm{X}$ is denoted by $P_{F}(X)$. In particular, when $\mathrm{X}=\mathrm{R}$, fuzzy points are also called fuzzy scalars and the set of all fuzzy scalars is denoted by $S_{F}(R)$. A fuzzy set A can be regarded as a set of fuzzy points belonging to it, i.e.

$$
A=\{(x, \lambda): A(x) \geq \lambda\} \quad \text { Or a set of fuzzy points on it } A=\{(x, \lambda): A(x)=\lambda\}
$$

\section{Fuzzy Metric Spaces}

3.1 FUZZY SCALARS: Suppose $(\mathrm{x}, \lambda)$ and $(\mathrm{y}, \lambda)$ are two fuzzy scalars. The following conditions are satisfied (a) We say (a, $\lambda) \succeq$ (b, $\gamma)$, if $a>b$ or $(\mathrm{a}, \lambda)=(\mathrm{b}, \gamma)$.

(b) (a, $\lambda$ ), is not less than (b, $\gamma$ ), if a $\geq$ b, denoted by (a, $\lambda) \succ$ (b, $\gamma$ ), or (a, $\lambda$ ) $\prec$ (b, $\gamma$ )

(c) $(\mathrm{a}, \lambda)$ is said to be non-negative if $a \geq 0$. The set of all non-negative fuzzy scalars is denoted by $S_{F}^{+}(R)$.

The definition (a) and (b) are both partial orders. When $\mathrm{R}$ is a subset of $S_{F}(R),(\mathrm{R}, \succ)$, and (R, $\left.\succeq\right)$, are the same as $(\mathrm{R}, \geq)$. Thus both $\succ$ and $\succeq$ can be viewed as some kind of generalization of the ordinary complete order $\geq$. It is obvious that the order defined in (a) is stronger than defined in (b).

3.2 FUZZY METRIC SPACE: Suppose $X$ is a non-empty set and $d_{F}: P_{F}(X) \cdot P_{F}(X) \rightarrow S_{F}^{+}(R)$, is a mapping. $\left(P_{F}(X), d_{F}\right)$ is said to be a fuzzy metric space if for any $(\mathrm{x}, \lambda),(\mathrm{y}, \gamma)$, and $(\mathrm{z}, \rho) \subset P_{F}(X), d_{F}$ satisfy the following three conditions. 
(i) $d_{F}((\mathrm{x}, \lambda),(\mathrm{y}, \gamma))=0$, iff $\mathrm{x}=\mathrm{y}$, and $\lambda=\gamma=1$

(ii) $\quad d_{F}((\mathrm{x}, \lambda),(\mathrm{y}, \gamma))=d_{F}((\mathrm{y}, \gamma),(\mathrm{x}, \lambda)) \quad($ Symmetric $)$

(iii) $\quad d_{F}((x, \lambda),(z, \rho)) \prec d_{F}((x, \lambda)+(y, \gamma))+d_{F}((y, \gamma)+(z, \rho))$, Triangular inequality

$d_{F}$ is called a fuzzy metric defined in $P_{F}(X)$, and $d_{F}((x, \lambda),(y, \gamma))$ is called fuzzy distance between two fuzzy points.

Note that fuzzy metric spaces have fuzzy points as their elements, ie they are sets of fuzzy points.

EXAMPLE :1 Suppose $(X, d)$ is an ordinary metric space. The distance of any two fuzzy points $(x, \lambda),(y, \gamma)$ in $P_{F}(X)$, is defined as

$$
d_{F}((x, \lambda),(y, \gamma)=\{d(x, y), \min (\lambda, \gamma)\}
$$

Where $d(x, y)$, is the distance between $\mathrm{x}$ and $\mathrm{y}$ defined in $(\mathrm{x}, \mathrm{d})$. Then $\left(P_{F}(X), d_{F}\right)$, is a fuzzy metric space.

PROOF : It suffices to prove that $d_{F}$ satisfied all the condition as defined in 3.2. Suppose that $(x, \lambda),(y, \gamma)$, are two fuzzy points in $P_{F}(X)$. Since $d(x, y)$ is the distance between $\mathrm{x}$ and y also $d(x, y) \geq 0$, if $x \neq y$, it follows from definition (1) that $d_{F}((x, \lambda),(y, \gamma))=\{d(x, y), \min (\lambda, \gamma)\}$ is non-negative fuzzy scalars. It is obvious that $d((x, \lambda),(y, \gamma))=0$, iff $d(x, y)=0$ and $\min \{\lambda, \gamma\}=1$, which is equal to that $x=y$, and $\lambda=\gamma=1$.

Symmetric: For any $\{(x, \lambda),(y, \gamma)\} \subset P_{F}(X)$, we have

$$
\begin{aligned}
& d_{F}((x, \lambda),(y, \gamma))=\{d(x, y) \min (\lambda, \gamma)\} \\
& d_{F}((x, \lambda),(y, \gamma))=\{d(y, x), \min (\gamma, \lambda)\} \\
& d_{F}((x, \lambda),(y, \gamma))=d_{F}((y, \gamma),(x, \lambda))
\end{aligned}
$$

Triangular inequality: For any $\{(x, \lambda),(y, \gamma),(z, \rho)\} \subset P_{F}(X)$, we have

$$
\begin{aligned}
d_{F}((x, \lambda),(z, \rho)) & =\{d(x, z), \min (\lambda, \rho)\} \\
d_{F}((x, \lambda),(z, \rho)) & \prec\{d(x, y)+(y, z)), \min (\lambda, \gamma, \rho)\} \\
& =\{d(x, y), \min (\lambda, \gamma)\}+\{d(y, z)), \min (\gamma, \rho)\} \\
& =d_{F}((x, \lambda),(y, \gamma))+d_{F}((y, \gamma),(z, \rho))
\end{aligned}
$$

3.3 DEFINITION: Suppose $\mathrm{X}$ is a non-empty set and $d_{F}: P_{F}(X) \cdot P_{F}(X) \rightarrow S_{F}^{+}(R)$, is a mapping. Then $\left(P_{F}(X), d_{F}\right)$,is said to be a strong fuzzy metric space, if it satisfies the first two condition in definition 3.2, and for any $(x, \lambda),(y, \gamma),(z, \rho)$,in $P_{F}(X)$ one has

(iii) $\quad d_{F}((x, \lambda),(z, \rho)) \preceq d_{F}((x, \lambda),(y, \gamma))+d_{F}((y, \gamma),(z, \rho))$

It is obvious from definition (3.2) and (3.3) that every strong fuzzy metric space is a fuzzy metric space. The following example shows the existence of strong fuzzy metric spaces and the difference between these two kinds of spaces.

EXAMPLE 2: Let L is a fuzzy linear space defined in $R^{n}$. The distance between arbitrary two fuzzy points $(x, \lambda)$ and $(y, \gamma)$ on $\mathrm{L}$ is defined by

$$
d_{F E}((x, \lambda),(y, \gamma))=\left\{d_{E}(x, y), \min (\lambda, \gamma)\right\}
$$

Where $d_{E}$ is the Euclidean distance. Then $\left(L, d_{F E}\right)$ is a strong fuzzy metric space where $\mathrm{L}$ denotes the set of fuzzy points on the fuzzy set L.

Proof : The first two condition can be proved just as example.1. Here we only prove the third one. Given arbitrary three fuzzy points on $\mathrm{L},(x, \lambda),(y, \gamma)$ and $(z, \rho)$.Since $\left(R^{n}, d_{E}\right)$ is a metric space.

$$
d_{E}(x, z) \leq d_{E}(x, y)+d_{E}(y, z)
$$


In the case of that inequality (ii) holds strictly, it is obvious from the definition 3.1(a) that the condition (iii)' is satisfied. In the other case, there must exists some $\lambda \in F$ such that $y=(1-\lambda) x+\lambda z$. Let $\alpha=\min \{\lambda, \rho\}$. We have that $\{x, z\} \subset L_{\alpha}$. Since $\mathrm{L}$ is a fuzzy linear space. $L_{\alpha}$ is a linear subspace of $R^{n}$ ( see the representation theorem of fuzzy linear space due to Lowen (1980) [13]). It follows that $y \in L_{\alpha}$ i.e $\gamma=L(y) \geq \alpha=\min \{\lambda, \rho\}$. This implies that $\min \{\lambda, \gamma, \rho\}=\min \{\lambda, \rho\}$.Thus one has

$$
\begin{aligned}
d_{F E}((x, \lambda),(z, \rho)) & =\left\{d_{E}(x, z), \min \{\lambda, \rho\}\right\} \\
& =\left(d_{E}(x, y)+d_{E}(y, z), \min \{\lambda, \gamma, \rho\}\right) \\
& =d_{F E}\left((x, \lambda),(y, \gamma)+d_{F E}((y, \gamma),(z, \rho))\right.
\end{aligned}
$$

Consequently, condition (iii)' is satisfied.

Note that the strong fuzzy metric space given above is a set of fuzzy points on the same fuzzy linear space. Different from it, the fuzzy metric space in example 2 comprises fuzzy points belonging to a fuzzy linear space. The difference is caused by that ' $\prec$ 'is replaced by the partial order ' $\preceq$ ' which is must stronger than it.

\section{The Completeness of Fuzzy Metric Spaces}

DEFINITION 4.1: let be $\left\{\left(a_{n}, \lambda_{n}\right)\right\}$ a sequence of fuzzy scalars. It is said to be convergent to a fuzzy scalars $(a, \lambda), \lambda \neq 0$ denoted by, $\lim _{n \rightarrow \infty}\left(a_{n}, \lambda_{n}\right) \rightarrow(a, \lambda)$ if $\lim _{n \rightarrow \infty} a_{n}=a,\left\{i \mid \lambda_{i}<\lambda, i \in N\right\}$ is a finite set and there exists a subsequence of $\left\{\lambda_{i}\right\}$, denoted by $\left\{\lambda_{l}\right\}$ such that $\lim _{n \rightarrow \infty} \lambda_{l}=\lambda$

DEFINITION 4.2: Suppose $\left(P_{F}(X), d_{F}\right)$ is the induced fuzzy metric space of $(X, d)$ and $\left\{\left(x_{n}, \lambda_{n}\right)\right\}$ is a sequence of fuzzy points in $\left(P_{F}(X), d_{F}\right) .\left\{\left(x_{n}, \lambda_{n}\right)\right\}$ is said to be convergent to a fuzzy points $(x, \lambda)$, if $\lim _{n \rightarrow \infty} d_{F}\left(\left(x_{n}, \lambda_{n}\right),(x, \lambda)\right)=0_{\lambda}$ and for any $\gamma \in(0,1]$ such that $\lim _{n \rightarrow \infty} d_{F}\left(\left(x_{n}, \lambda_{n}\right),(x, \gamma)\right)=0_{\gamma} \quad$ one has $\lambda \geq \gamma,(x, \lambda)$ is called the limit of the sequence denoted by. $\lim _{n \rightarrow \infty}\left(x_{n}, \lambda_{n}\right)=(x, \lambda)$

PROPOSITION 4.1: Suppose $\left\{\left(x_{n}, \lambda_{n}\right)\right\}$ is a sequence of fuzzy points in $\left(P_{F}(X), d_{F}\right)$ and $(x, \lambda) \in$ $\left(P_{F}(X), d_{F}\right), \lambda \neq 0$. We have that $\lim _{n \rightarrow \infty}\left(x_{n}, \lambda_{n}\right)=(x, \lambda)$ if and only if, $\lim _{n \rightarrow \infty} x_{n}=x\left\{i \mid \lambda_{i}<\lambda, i \in N\right\}$ is a finite set and there exists a subsequence $\left\{\lambda_{i}\right\}$, denoted by $\left\{\lambda_{l}\right\}$ such that $\lim _{n \rightarrow \infty} \lambda_{l}=\lambda$.

PROOF: It is obvious.

DEFINITION 4.3: A sequence of fuzzy points $\left(x_{n}, \lambda_{n}\right) \in\left(P_{F}(X), d_{F}\right)$ is said to be a Cauchy sequence if there exists some $\lambda \in(0,1]$ such that $\lim _{n \rightarrow \infty} d_{F}\left(\left(x_{m+n}, \lambda_{m+n}\right),\left(x_{n}, \lambda_{n}\right)=0_{\lambda}\right.$, for all $m \in N$.

Note that every Cauchy sequence of fuzzy points defined above has a unique fuzzy point as its limit, which is very similar to classic one.

DEFINITION 4.4: An induced fuzzy metric space is said to be complete if any Cauchy sequence in it has a unique limit in the space.

THEOREM 4.2: Suppose $\left(P_{F}(X), d_{F}\right)$ is the induced fuzzy metric space of an ordinary metric space $(X, d)$

. Then it is complete iff $(X, d)$ is complete.

PROOF : Necessary condition - It is obvious.

Sufficient condition: Suppose $\left\{\left(x_{n}, \lambda_{n}\right)\right\}$ is an arbitrary Cauchy sequence of $\left(P_{F}(X), d_{F}\right)$. Since $(X, d)$ is complete and, $\lim _{n \rightarrow \infty} d\left(x_{m+n}, x_{n}\right)=0$ for any $m \in N$ denote the index set $\left\{l \mid \lambda_{l}=\min \left\{\lambda_{m+n}, \lambda_{n}\right\}, n=1,2, \ldots.\right\}$ by $L_{m}$. From the definition of Cauchy sequences of fuzzy points, there exists some $\lambda \in(0,1]$ such that for any $m \in N$, the set $\left\{l \mid \lambda_{l}<\lambda, l \in L_{m}\right\}$ is finite and there exists a 
subsequence of $\left\{\lambda_{l}\right\}_{l \in L_{m}}$, denoted by $\left\{\lambda_{k}\right\}$ which is also a subsequence of $\left\{\lambda_{n}\right\}$,such that. $\lim _{k \rightarrow \infty} \lambda_{k}=\lambda$ It is obvious that $\left\{l \mid \lambda_{l}<\lambda, l \in L_{m}\right\} \supset\left\{n \mid \lambda_{n}<\lambda, n=1,2, \ldots ..\right\}$.

Consequently, $\left\{n \mid \lambda_{n}<\lambda, n=1,2, \ldots ..\right\}$, is also a finite set. From the above arguments, we have $\lim _{n \rightarrow \infty}\left(x_{n}, \lambda_{n}\right)=(x, \lambda)$. It implies that there exists a limit of $\left\{x_{n}, \lambda_{n}\right\}$ in $P_{F}(X)$. In the following, we prove the uniqueness, by contradiction, assume that there is another limit of the same Cauchy sequence $\left\{\left(x_{n}, \lambda_{n}\right)\right\}$. Since we know that ' $x$ ' is unique limit of $\left\{x_{n}\right\}$, we can denote by $(x, \gamma)$ the limit different from $(x, \lambda)$, $\gamma \neq \lambda$, say $\gamma>\lambda$. Then we have $\left\{n \mid \lambda_{n}<\gamma\right\}$ is a finite set. From the above arguments, we know that, $\lim _{k \rightarrow \infty} \lambda_{k}=\lambda$ and $\left\{k \mid \lambda_{k}<\lambda\right\}$ is finite set. Thus taking $\rho=\frac{\lambda+\gamma}{2}$ we have $\left\{\lambda_{k}\right\} \cap\{\lambda, \rho\} \subset\left\{n \mid \lambda_{n}<\gamma\right\}$ , is an infinite set. This contradicts that $\left\{n \mid \lambda_{n}<\gamma\right\}$ is finite set. Therefore there is only one limit of Cauchy sequence.

Note that the strong fuzzy linear metric space is generally not complete. It can be seen through the example given below.

EXAMPLE 3: Consider the strong fuzzy linear metric space $\left(L, d_{F E}\right)$, where

$$
L=\left\{(x, \lambda) \mid x \in R \backslash\{0\} . \lambda=\frac{1}{2}\right\} \cup\{(0,1)\}
$$

And $d_{F E}$ is induced by the ordinary Euclidean metric $d_{E}$. The sequence $\left\{\left(\frac{1}{n}, \frac{1}{2}\right)\right\}$ in $\mathrm{L}$ is a Cauchy sequence in the sense of definition 4.3. However, the limit of the sequence, $(0,1 / 2)$ is not on the space $\mathrm{L}$.

\section{Main Results}

Definition 5.1: Let $\left(P_{F}(X), d_{F}\right)$ is the induced fuzzy metric space of $(X, d)$. If $\phi$ is a mapping from $P_{F}(X)$ into $P_{F}(X)$,and let $c<1$ be a number such that

$$
d(\phi(x, \lambda), \phi(y, \gamma)) \leq c\{d(x, y), \min (\lambda, \gamma)\}
$$

For all $\{(x, \lambda),(y, \gamma)\} \subset P_{F}(X)$, then $\phi$ is said to be a contraction of $P_{F}(X)$ into $P_{F}(X)$.

THEOREM 5.1: If $\left(P_{F}(X), d_{F}\right)$ is the induced fuzzy metric space of complete metric space $(X, d)$ and if $\phi$ is a contraction of $P_{F}(X)$ into $P_{F}(X)$, then there exists one and only one $(x, \lambda)$ of $P_{F}(X)$ such that $\phi(x, \lambda)=(x, \lambda)$.

PROOF : Let $\left(x_{0}, \lambda_{0}\right) \subset P_{F}(X)$, be arbitrary and define $\left\{\left(x_{n}, \lambda_{n}\right)\right\}$ be a sequence of fuzzy scalars by setting $\left(x_{n+1}, \lambda_{n+1}\right)=\phi\left(x_{n}, \lambda_{n}\right), \quad n=0,1,2$.

Choose $c<1$, so that

$d_{F}(\phi(x, \lambda), \phi(y, \gamma)) \leq c\{d(x, y), \min (\lambda, \gamma)\}$, holds for $n \geq 1$, then we have

$d_{F}\left(\left(x_{n+1}, \lambda_{n+1}\right),\left(y_{n}, \gamma_{n}\right)\right)=d_{F}\left(\phi\left(x_{n}, \lambda_{n}\right), \phi\left(y_{n-1}, \gamma_{n-1}\right)\right)$

$d_{F}\left(\left(x_{n+1}, \lambda_{n+1}\right),\left(y_{n}, \gamma_{n}\right)\right) \leq c\left(d\left(x_{n}, y_{n-1}\right), \min \left(\lambda_{n}, \gamma_{n-1}\right)\right)$

By induction we have,

$$
d_{F}\left(\left(x_{n+1}, \lambda_{n+1}\right),\left(y_{n}, \gamma_{n}\right)\right) \leq c^{n}\left\{d\left(x_{1}, y_{0}\right) \min \left(\lambda_{1}, \gamma_{0}\right)\right\}, \quad n=0,1,2, \ldots \ldots \ldots
$$

Since $\left(P_{F}(X), d_{F}\right)$, is induced fuzzy metric space of complete metric $(X, d)$, hence by theorem (4.2), the $\left(P_{F}(X), d_{F}\right)$ is complete fuzzy metric space. Therefore the sequence $\left\{\left(x_{n}, \lambda_{n}\right)\right\}$ of fuzzy points in 
$\left(P_{F}(X), d_{F}\right)$, is said to be convergent to fuzzy point $(x, \lambda)$ if $\lim _{n \rightarrow \infty}\left(\left(x_{n}, \lambda_{n}\right),(x, \lambda)\right)=0_{\lambda}$, and for any $\gamma \in(0,1]$ such that $d_{F}\left(\left(x_{n}, \lambda_{n}\right),(x, \gamma)\right)=0_{\gamma}$,one has $\lambda \geq \gamma,(x, \lambda)$ is called the limit of the sequence denoted by $\lim _{n \rightarrow \infty}\left\{\left(x_{n}, \lambda_{n}\right)\right\}=(x, \lambda)$, If $n<m$, it follow that

$$
\begin{aligned}
& d_{F}\left(\left(x_{n}, \lambda_{n}\right),\left(y_{m}, \gamma_{m}\right)\right) \leq \sum_{i=n+1}^{m} d_{F}\left(\phi\left(x_{i}, \lambda_{i}\right), \phi\left(y_{i-1}, \gamma_{i-1}\right)\right) \\
& d_{F}\left(\left(x_{n}, \lambda_{n}\right),\left(y_{m}, \gamma_{m}\right)\right) \leq\left(c^{n}+c^{n+1}+\ldots \ldots+c^{m-1}\right) d\left(\left(x_{1}, y_{0}\right), \min \left(\lambda_{1}, \gamma_{0}\right)\right)
\end{aligned}
$$

Since $\left\{\left(x_{n}, \lambda_{n}\right)\right\}$,is a Cauchy sequence in $\left(P_{F}(X), d_{F}\right)$, and it is complete, hence

$\lim _{n \rightarrow \infty}\left\{\left(x_{n}, \lambda_{n}\right)\right\}=(x, \lambda)$, for some $(x, \lambda) \subset P_{F}(X)$

Since $\phi$ is contraction and it is continuous in $P_{F}(X)$, i.e

$$
\phi(x, \lambda)=\lim _{n \rightarrow \infty} \phi\left(x_{n}, \lambda_{n}\right)=\lim _{n \rightarrow \infty}\left\{\left(x_{n+1}, \lambda_{n+1}\right)\right\}=(x, \lambda)
$$

Hence the theorem.

\section{References}

[1]. B.B.Chaudhary and A.Rosenfeld, “On a metric distance between fuzzy sets," Pattern Recognition Letters 17(1996),1157 - 1160.

[2]. P.Diamond and P.Kloden,"Metric Spaces of fuzzy sets, Fuzzy sets and system 35(1990),241-249.

[3]. A.George and P.V.Veeramani,"On some results of fuzzy metric spaces," Fuzzy sets and system 64(1994),395 - 399.

[4]. A.George and P.V.Veeramani, "on some results of analysis for fuzzy metric spaces" Fuzzy sets and systems.90(1997),365 - 368.

[5]. Z.Q.Xia and F.F.Guo,’Fuzzy metric spaces” J. Appl. Math.\& Computing, Vol-16(2004),No.1-2,371-381.

[6]. O.Kaleva and Seikkala, "On fuzzy metric space” Fuzzy sets and systems. 12(1984),215-229

[7]. R.Lowen,"Convex fuzzy sets", Fuzzy sets and systems.3 (1980),291-310.

[8]. B.M.Pu and Y.M.Liu,'Fuzzy topology I" Neighbourhood structure of a fuzzy point and Moore-smith convergence, J.Math.Anal.Appl.76(1980),517-599.

[9]. Rudin,Walter "Principles of mathematical analysis" McGRAW-HILL KOGAKUSHA LTD.3 ${ }^{\text {rd }}$ Edition 1976.

[10]. V.Gregori and S.Romaguera,’Some Properties of fuzzy metric spaces” Fuzzy sets and systems.115(2000),485-489. 